\title{
Primary Cerebellar Neuroendocrine Tumors: Chimeras or Real Entities? A Case Report with a 6-Year Follow-Up
}

\author{
Claudio Vernieri ${ }^{\mathrm{a}, \mathrm{b}} \quad$ Daniela Femia $^{\mathrm{a}} \quad$ Sara Pusceddu $^{\mathrm{a}}$ Carlo Capella $^{\mathrm{a}}$ \\ Juan Rosai ${ }^{a}$ Giuseppina Calareso $^{a}$ Laura Concas $^{a}$ Natalie Prinzi ${ }^{a}$ \\ Giuseppe Lo Russo $^{\mathrm{a}} \quad$ Filippo de Braud $^{\mathrm{a}} \quad$ Roberto Buzzoni ${ }^{\mathrm{a}}$ \\ ${ }^{a}$ Istituto Nazionale Tumori, Milan, Italy; ${ }^{b}$ IFOM - Istituto FIRC di oncologia molecolare, \\ Milan, Italy
}

\section{Keywords}

Brain tumor - Radiotherapy · Surgery · Well differentiated neuroendocrine tumor - Cerebellum

\begin{abstract}
We report the case of a 38-year-old patient who was diagnosed with a cerebellar welldifferentiated neuroendocrine tumor (WDNET) in 2009. At first glance, we believed that it was a metastasis from an unrecognized WDNET arising outside the cerebellum. However, despite a prolonged follow-up of 6 years, an extracranial WDNET has never been found. During this time, the tumor recurred locally twice, and the patient was treated with surgery and radiotherapy. At the moment, he enjoys good general conditions and his tumor is under control. Due to the histopathological characteristics and clinical behavior of the tumor, we believe that this is the first report to date of a primary cerebellar WDNET.
\end{abstract}

\footnotetext{
(C) 2016 The Author(s)

Published by S. Karger AG, Basel
}

\section{Introduction}

Primary well-differentiated neuroendocrine tumors (WDNETs) arising in the intracranial portion of the central nervous system (CNS) are occasional findings. To date, less than 


\section{Case Reports in Oncology}

10 cases of CNS WDNETs have been reported, 3 of which originated from cerebral hemispheres or the skull base and another 3 were paragangliomas [1-6].

On the other hand, the CNS more frequently represents a metastatic site for primary poorly differentiated NET arising elsewhere, most often in the lung (small-cell lung cancer) $[7,8]$. In such cases, other secondary lesions, such as liver, lung and lymph nodes metastases, are often detected synchronously or metachronously with CNS localizations.

Patients with WDNET and brain metastases are rare; typically they have a poor prognosis, with one study reporting a median survival time of 10.0 months after diagnosis of brain metastases [7]. Overall, brain metastases occur in 1.5-6\% of all WDNETs, and their detection is usually preceded by the documentation of the primary, extracranial tumor $[7,8]$.

\section{Case Report}

In August 2009, a 38-year-old man referred to the hospital emergency department after a 2-month history of vertigos. Anamnesis and physical examination, including neurological evaluation, were not suggestive of any specific disease. Magnetic resonance (MR) imaging of the brain was performed which identified a large, heterogeneous contrast-enhancing lesion in the left cerebellar hemisphere, surrounded by significant edema (fig. 1, red arrows). Images were suspicious for a primary CNS neoplasm. Therefore, and given the potential risk for serious complications, immediate radical neurosurgery was performed, without previous systemic staging of the disease. Histology of the surgical specimen was compatible with a grade 2 (G2), WDNET localization with vaguely lobular architecture and sporadic small clusters of foamy macrophages. Lobules of neoplastic cells were separated by a thin network of fibrous septa enriched in vessels with a few aggregates of mature lymphocytes within them. Neoplastic cells contained polygonal, eosinophilic cytoplasm and ovoid nuclei with irregular profiles, coarse chromatin and one or two eosinophilic nucleoli (fig. 2a, b). Several cells exhibited finely vacuolar cytoplasm and a few rhabdoid cells were also present. Brisk mitotic activity was detected, with Ki67/MIB-1 staining positive in around 15-20\% of tumor cells (fig. 2c). At immunohistochemistry, neoplastic cells diffusely expressed synaptophysin, chromogranin A (CgA) (fig. 2d) and neuron-specific enolase (NSE). EGFR was expressed on the cytoplasmic membrane of about $30 \%$ cells, while vimentin was detectable in the few rhabdoid cells. p53, EMA, cytokeratin CAM5.2, cytokeratin 7 and CD10 were expressed only in a few neoplastic cells, while staining was consistently negative for Neu-N, Oligo-1, myogenin, TTF-1, PSA, smooth muscle actin, Melan-A, Mart-1, S-100 protein, CD31, somatostatin receptor, cytokeratins AE1/AE3, GFAP and progesterone receptors.

Then the patient came to our attention. Given the peculiarity of the case, we asked two other pathologists with proven experience in the field of NET to review the slides. The morphological and immunohistochemical (IHC) analysis showed diffuse positive staining for the neuroendocrine markers such as synaptophysin, CgA and NSE, with a focal staining for cytokeratins 7 and 8/18. Glioma markers (GFAP, S-100), calcitonin, pituitary (ACTH, FSH, GH, LH, PRL, TSH, alpha-SU), and gastroenteropancreatic and pulmonary NET hormones (serotonin, gastrin, insulin, somatostatin, PP, glucagon/glycentin, CDX2, PDX1, TTF1) were instead undetectable. A plausible differential diagnosis with Pineocytomas with mixed/intermediate differentiation (PPT) was proposed because PPT cells often assemble in lobular structures and stain positive for CgA, NSE and synaptophysin [9]. However, the absence of pineal localization in our case, and the cytological coarse chromatin aspect, was clearly dif- 


\section{Case Reports in Oncology}

Vernieri et al.: Primary Cerebellar Neuroendocrine Tumors: Chimeras or Real Entities? A Case Report with a 6-Year Follow-Up

ferent from the typically stippled pattern observed in PPTs [9]. Both pathologists confirmed the diagnosis of G2 WDNET according to the criteria of the 2010 WHO classification [10].

Only one ectopic cerebellar localization of a presumptive, primary pineal tumor has been reported in the literature; in that case, the primary tumor consisted of an immature dysembryoma [11]. For these reasons, pathologists excluded the hypothesis of an ectopic PPT.

Given that primary cerebellar WDNETs have never been described to date, the possibility of a brain metastasis from an elsewhere localized WDNET was considered reliable. However, extensive imaging studies, including computed tomography (CT), ${ }^{68} \mathrm{Ga}$ PET and ${ }^{18} \mathrm{FDG}-$ PET, did not show any evidence of extracranial primary tumor. Despite this, we expected that a primary NET located outside the brain, or other organ metastases, would become evident over a prolonged follow-up, also since no systemic treatment was administered to inhibit the growth of potentially present extracerebral tumor cells. However, total-body radiological studies including brain CT and MR imaging regularly performed every 6 months proved negative, and the patient remained asymptomatic and in good general conditions, without evidence of other extracerebral localizations of the disease.

In October 2012, vertigos reappeared and a brain MRI examination documented one local recurrence of the previously resected left hemisphere cerebellar lesion and the appearance of a new small lesion in maximum diameter, located at the left cerebellopontine angle (fig. 3, red arrows). Extensive systemic staging including ${ }^{68} \mathrm{Ga}$ PET was performed, but again no extracranial localizations were detected. Partial surgical resection of the cerebellopontine lesion was then performed, followed by stereotactic radiotherapy on residual disease (60 Gy) and on left cerebellar hemisphere (50 Gy). Pathological and IHC examination of the recurrence confirmed the primary diagnosis.

In June 2014, another cerebellar recurrence was treated with radiotherapy (50 Gy). The patient also underwent total-spine MR imaging and cytological evaluation of cerebrospinal fluid, none of which showed any evidence of disease. At the last control in July 2015, minimal disease persistence at the cerebellopontine angle $(13 \times 6 \times 9 \mathrm{~mm})$ was documented by brain MRI, with the patient remaining substantially asymptomatic and involved in normal daily and working activities. Despite biannual systemic re-evaluations with total-body contrastenhanced CT and ${ }^{68} \mathrm{Ga}$ PET, according to the clinical course of SNC primary tumors, extracranial disease has never become evident over a total follow-up of 6 years.

\section{Discussion}

In $10-13 \%$ of metastatic WDNETs, the primary tumor cannot be identified at diagnosis and can even remain occult during the entire course of the disease, with the liver being the main site of metastatic spread in such cases [12]. This can be explained by three different hypotheses: (a) if clinical follow-up is long enough, the primary tumor eventually becomes detectable; (b) the primary lesion remains small or even spontaneously regresses due to poor vascularization or clearing by the immune system; (c) the primary tumor arises in the same organ that is erroneously considered the metastatic site (in most cases the liver) [13].

The case described here unlikely represents a metastatic-to-the-cerebellum WDNET of unknown primary origin because of the following reasons.

(1) The putative primary extracranial tumor has not been detected during a prolonged, 6-year-long follow-up. This strongly contrasts with data in the literature, where detection of 
detection of the primary neoplasm, most often at the lungs [7, 8].

(2) Tumor recurrences occurred in the same area of the brain as the first detected lesion, whereas lesions in sites that are preferential metastatic localizations of extracranial WDNETs, such as the liver and thoracic/abdominal lymph nodes, have never been found.

(3) Although IHC markers of WDNETs are not absolutely specific of any anatomic origin, the lack of TTF1, CDX2 and PDX1 expression in the tumor makes it unlikely to originate from the lung, gut and pancreas [14], thus probably excluding the most frequent sites of NET origin.

(4) The clinical indolent course of the patient, characterized by long-term survival, is typical of advanced WDNETs.

(5) Although it cannot be definitively excluded that a small, primary extracranial NET has metastasized to the brain before becoming quiescent or even being cleared by the immune system, it is hard to believe that the liver, lung and lymph nodes were completely spared, with the cerebellum instead being the only site of subsequent metastatic waves. (MB).

(6) Plausible differential diagnoses, including PPT and cerebellar medulloblastoma

We therefore conclude that this case represents the first pathological report of a primary cerebellar WDNET. Recurrences observed during patient follow-up represent, in our opinion, locoregional relapses of the primary CNS tumor. This work aims, above all, to make aware pathologists and clinicians about the existence of primary WDNETs of the CNS. These tumors would definitively consist of very rare entities, neglected until now because erroneously considered metastatic localizations of elsewhere located primary neuroendocrine neoplasms. They could originate from sparse cerebellar cells of the diffuse neuroendocrine system.

\section{Acknowledgements}

Editorial assistance was provided by Luca Giacomelli, $\mathrm{PhD}$, and Lilia Biscaglia, $\mathrm{PhD}$; this assistance was supported by internal funds.

\section{Statement of Ethics}

Written informed consent was obtained from the patient for publication of this case report and any accompanying images.

\section{Disclosure Statement}

The authors declare that there is no conflict of interest that could be perceived as prejudicing the impartiality of the case report.

This case report did not receive any specific grant from any funding agency in the public, commercial or not-for-profit sector. 


\section{References}

1 Deshaies EM, Adamo MA, Qian J, DiRisio DA: A carcinoid tumor mimicking an isolated intracranial meningioma. Case report. J Neurosurg 2004;101:858-860.

2 Ibrahim M, Yousef M, Bohnen N, Eisbruch A, Parmar H: Primary carcinoid tumor of the skull base: case report and review of the literature. J Neuroimaging 2010;20:390-392.

-3 Tamura R, Kuroshima Y, Nakamura Y: Primary neuroendocrine tumor in brain. Case Rep Neurol Med 2014;2014:295253.

4 Prayson RA, Chahlavi A, Luciano M: Cerebellar paraganglioma. Ann Diagn Pathol 2004;8:219-223.

5 Deb P, Sharma MC, Gaikwad S, Gupta A, Mehta VS, Sarkar C: Cerebellopontine angle paraganglioma report of a case and review of literature. J Neurooncol 2005;74:65-69.

-6 Li ZJ, Lan XL, Hao FY, Yao WC, Wang MY, Chen XD, Bo YL, Wang JP, Zhang HD, Sun P: Primary cerebellar paraganglioma: a pediatric case report and review of the literature. Pediatr Neurol 2014;50:303-306. Hlatky R, Suki D, Sawaya R: Carcinoid metastasis to the brain. Cancer 2004;101:2605-2613.

8 Patchell RA, Posner JB: Neurologic complications of carcinoid. Neurology 1986;36:745-749.

$\$ 9$ Jouvet A, Saint-Pierre G, Fauchon F, Privat K, Bouffet E, Ruchoux MM, Chauveinc L, Fèvre-Montange M: Pineal parenchymal tumors: a correlation of histological features with prognosis in 66 cases. Brain Pathol 2000;10:49-60.

10 Bosman F, Carneiro F, Hruban R, Theise ND: WHO Classification of Tumors of the Digestive System. Lyon, IARC Press, 2010.

11 Steimle R, Raffi A, Bonneville JF, Carbillet JP, Schraub S, Jacquet G, Abdul-Razzak A: Germinoma (ectopic pinealoma) with double location: supra-sellar and the cerebellum without pineal tumor (in French). Neurochirurgie 1979;25:129-133.

12 Maxwell JE, Sherman SK, Stashek KM, O’Dorisio TM, Bellizzi AM, Howe JR: A pratical method to determine the site of unknown primary in metastatic neuroendocrine tumors. Surgery 2014;156:13591365.

13 Jessy T: Immunity over inability: The spontaneous regression of cancer. J Nat Sci Biol Med 2011;2:4349.

14 Srivastava A, Hornick JL: Immunohistochemical staining for CDX-2, PDX-1, NESP-55, and TTF-1 can help distinguish gastrointestinal carcinoid tumors from pancreatic endocrine and pulmonary carcinoid tumors. Am J Surg Pathol 2009;33:626-632. 


\section{Case Reports in Oncology}

\begin{tabular}{l|l}
\hline Case Rep Oncol 2016;9:432-439 \\
\hline $10.1159 / 000447771$ & $\begin{array}{l}\text { C 2016 The Author(s). Published by S. Karger AG, Basel } \\
\text { www.karger.com/cro }\end{array}$ \\
\hline
\end{tabular}

Vernieri et al.: Primary Cerebellar Neuroendocrine Tumors: Chimeras or Real Entities? A Case Report with a 6-Year Follow-Up
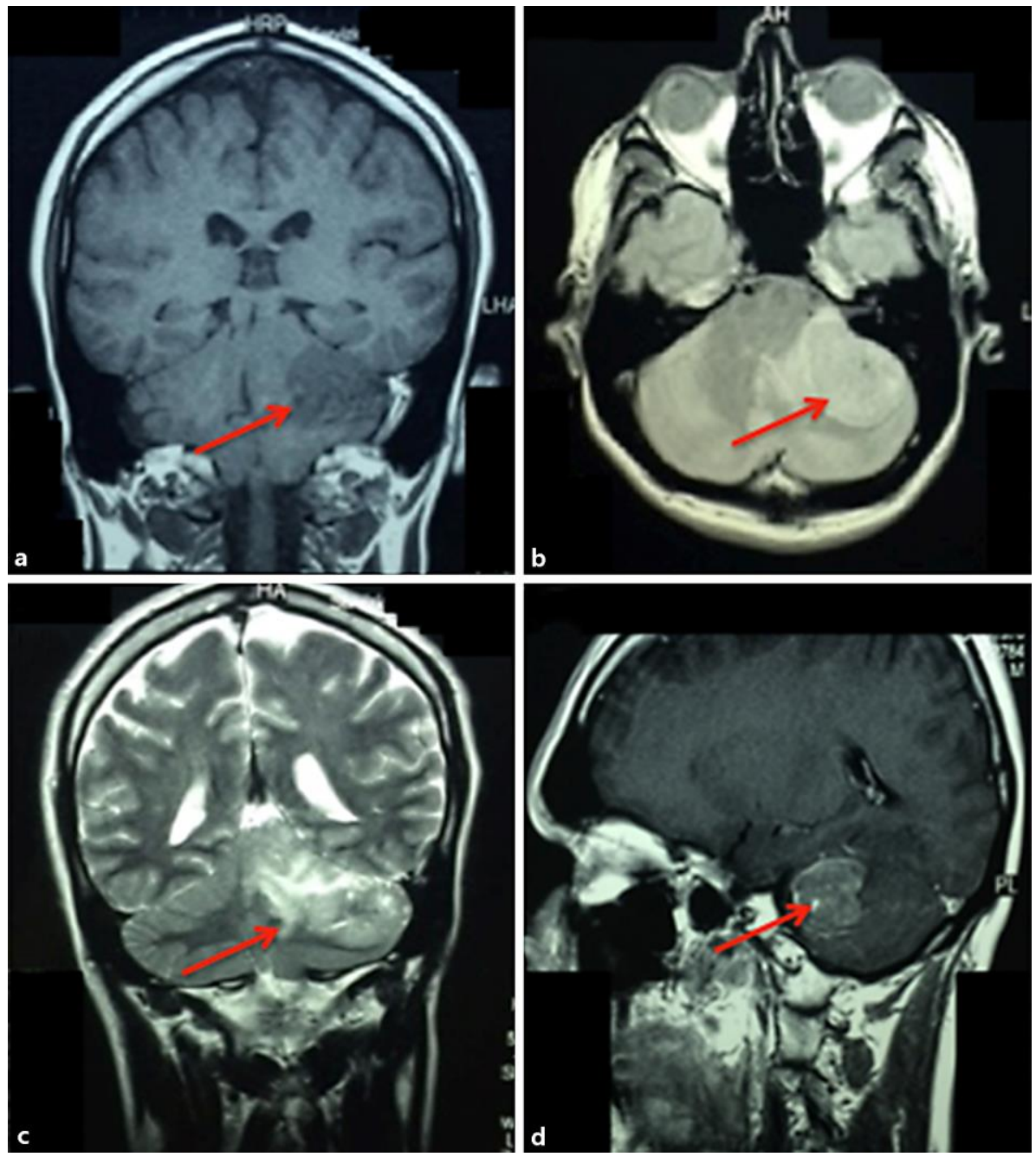

Fig. 1. a Coronal plane, nonenhanced T1-weighted MR scan. b Axial, FLARE sequence. c Coronal plane, T2weighted MR scan. d Sagittal plane, contrast-enhanced T1-weighted scan. 


\section{Case Reports in Oncology}
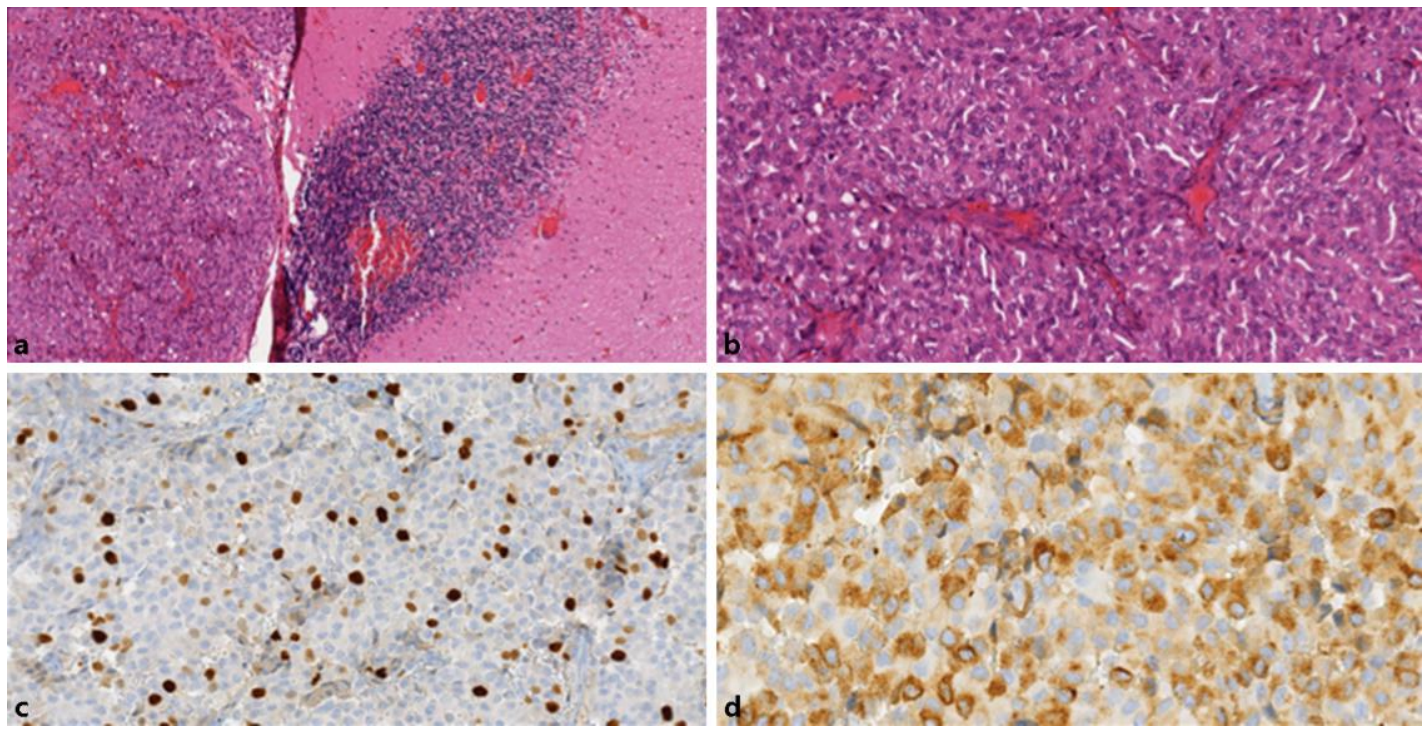

Fig. 2. Hematoxylin-eosin staining: $\times 10$ magnification (a), $\times 20$ magnification (b). c IHC for MIB1. d IHC for chromogranin A. 


\section{Case Reports in Oncology}

\begin{tabular}{l|l}
\hline Case Rep Oncol 2016;9:432-439 \\
\hline $10.1159 / 000447771$ & $\begin{array}{l}\text { ○ 2016 The Author(s). Published by S. Karger AG, Basel } \\
\text { www.karger.com/cro }\end{array}$ \\
\hline
\end{tabular}

Vernieri et al.: Primary Cerebellar Neuroendocrine Tumors: Chimeras or Real Entities? A Case Report with a 6-Year Follow-Up
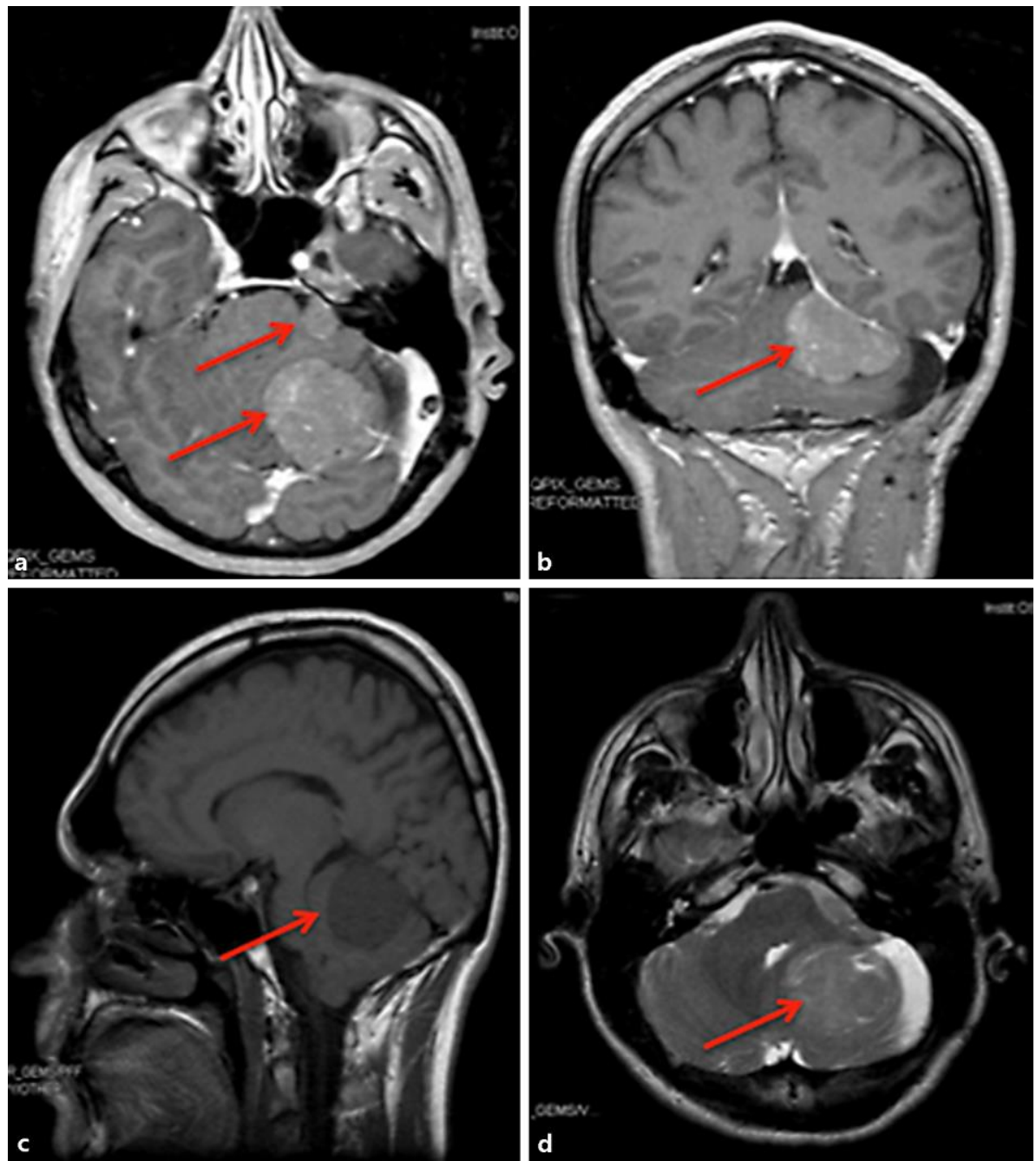

Fig. 3. a Axial, contrast-enhanced T1-weighted MR scan. b Coronal, contrast-enhanced T1 scan. c Sagittal plane, T1-weighted MR scan, no contrast. $\mathbf{d}$ Axial plane, FLAIR sequence. 